www.nature.com/ijo

\title{
PREFACE
}

\section{Dysfunctional foods in pathogenesis of obesity and metabolic syndrome}

International Journal of Obesity (2008) 32, S1-S3; doi:10.1038/ijo.2008.200

The publication of this 4th Fribourg Obesity Research Conference on the theme of 'dysfunctional foods' coincides with a recent proposal of the European Union to spend 90 million euros yearly to provide free fruits and vegetables to schools across the continent in a drive to curb childhood obesity. Whether this European Union proposal, if implemented, will prove to be a turning point in genuine political will to curtail the dramatic escalation of childhood obesity in European Union countries is far from certain. It nonetheless embodies an urgent need for reversing the increasing trend of children to consume - at the expense of fruits and vegetables unhealthy foods and snacks that are too fatty, too salty and too sugary.

\section{Early markers of risks for later metabolic syndrome}

Exposure to such unhealthy diets encourages overeating, and hence will put children at risk of the damaging metabolic and cardiovascular insults of excess fat gain during critical periods of growth and development. Furthermore, as discussed by Zimmerman and Aeberli in their review on the relationships between dietary factors and altered metabolism in overweight children, their higher intake of saturated fat and sugary drinks, and lower intake of antioxidants and vitamins, can each be linked to one or more specific risk factors for metabolic syndrome, such as insulin resistance, hypertension, dyslipidaemia and pro-inflammatory status.

\section{Household predispositions towards unhealthy foods and obesity}

Robinson and Godfrey point to the importance of shared family environment and dietary habits, and argue that since early life may be a time when dietary practices are established that will continue throughout childhood, there is a need to understand the evolution of dietary habits in early life and to determine how these practices influence obesity risk. They also address the notion that the mother's feeding habits during pregnancy and lactation may programme the fetus or neonate towards later obesity- a notion that is strongly supported by animal studies indicating that manipulation of maternal diet during pregnancy or lactation can lead to perturbations in appetite regulatory mechanisms and to obesity in the offspring. Furthermore, the recent demonstration in rats that offspring of mothers treated with leptin during lactation are protected from dietary-fat-induced obesity later in life raises pertinent questions as to the clinical relevance of these observations in the context that leptin is present in breast milk, but not in infant formulae, and that breast-feeding has been reported to lower the risk for later obesity compared with formula feeding. The ongoing debate about the role of breast-feeding versus bottle-feeding in susceptibility to obesity is further fuelled by another intriguing hypothesis that the obesogenic risks associated with infant formula may reside in the chemical composition of the milk bottle! This follows emerging evidence indicating that mice develop obesity if exposed during the fetal or neonatal period to endocrinedisrupting chemicals that mimic or alter the effects of hormones, some of which - such as perfluorooctanoic acid and tributyltin - are used to make baby bottles, the lining of food tins and some plastic food wraps and containers, as well as in scores of products ranging from microwave popcorn bags to pizza box liners and other food containers. In a review on this topic of endocrine disruptors, Casals-Casas, Feige and Desvergne highlight the possible involvement of a variety of these household endocrine-disrupting chemicals in disrupting normal developmental and homoeostatic controls over adipogenesis and energy balance, and discuss the molecular mechanisms by which they may predispose to obesity and sensitize exposed individuals to obesity and related metabolic disorders.

\section{Youth-targeted advertisements}

In addition to the potential role of diet and endocrinedisrupting chemicals in developmental programming and the role of parents as poor models for early eating behaviour, a strong association between the number of hours of television viewing and childhood obesity has repeatedly been shown in many countries. Besides displacing physical activity and encouraging passive snacking, television watching also seems to have a broader impact on food intake 
S2

through food advertisements and cross-promotion of food products and media characters. Television watching therefore provides the most powerful medium for advertisements geared at sustainable habit formation for energy-dense foods and sugar-sweetened soft drinks. That early habit formation and long-lasting learned behaviours is now under the control of 'neuro-marketing' strategies is discussed by Sonneville and Gortmaker within the context of a broader analysis of total energy intake and the energy gap (that is, the daily imbalance between energy intake and expenditure) associated with discretionary behaviours of adolescents. They predict that the relationship of video game playing and energy balance will also change over time, given the advent of new generation active video games and the increase of online gaming, which may be laden with targeted advertising. They also draw attention to the fact that advertisements for fast foods and sugary beverages are now entwined with physical activity and sports, and point out that if advertising messages have the power to influence energy intake in response to physical activity, and opportunities for ingesting fast foods are incorporated into practices and games, then increasing physical activity could be an unsuccessful approach for reducing positive energy balance.

\section{Unhealthy foods and pathogenesis of obesity and diseases}

How these fast foods promote positive energy balance seems to involve multiple pathways. Fats, sugars and salts certainly contribute enormously to the hedonic properties of food products, and hence stimulate appetite, whereas foods high in fats and sugars - by virtue of their high energy density and low bulk-produce less gastric distension and delay gastric emptying, thereby retarding the feeling of fullness and the cessation of eating. Once absorbed, the products of fatty foods may also interfere with appetite control mechanisms operating through nutrient sensing, and impair neurohormonal signals generated by specialized cells that detect variations in blood glucose or lipid concentrations. In his review focused on glucose sensors, Thorens explores first the physiological role of glucose as a signal that regulates body weight and blood glucose. He then argues the case that the mechanism of glucose sensing that controls insulin secretion by the pancreatic $\beta$-cells is also used by other cell types, and discusses how deregulation of these extra pancreatic glucose sensors, possibly because of lipid-induced dysfunction, may be early events in the pathogenesis of obesity and type 2 diabetes.

\section{Sugars, salt and alcohols}

Such interactions between nutrients and glucose sensors also raise the question as to whether fructose may also impact on glucose sensors and influence appetite. The use of highfructose corn syrup as a caloric sweetener in beverages is often blamed as an important contributing factor to the surge in childhood obesity - a contention that is fuelled by the hypothesis that fructose bypasses food-intake regulatory systems and favours lipogenesis, and that fructose, rather than glucose, is the more damaging sugar component in terms of risks for diabetes, atherosclerosis and hypertension. The recent advances on this topic of sugary drinks in the pathogenesis of obesity and cardiovascular diseases are reviewed by Brown et al., who also underscore the need to study the mechanisms of cardiovascular dysregulation induced by fructose in the context of its potential interactions with caffeine, which is often present in sugary soft drinks, as well as in the context of a link between sugary drinks and salt intake in children and adolescents.

Indeed, highly salted foods make children (and adults) thirsty, which amplifies the likelihood for greater sugary drink consumption. Furthermore, the propensity of obese individuals to hypertension and other cardiovascular risks will be amplified if they are on a high salt intake-a common feature because of the obese individual's higher absolute intake. How dietary salt (or sodium) affects blood pressure is discussed by Van Vliet and Montani with special emphasis on the time course of salt-induced hypertension. They bring forward compelling evidence that salt affects blood pressure in at least two distinct ways, one being a relatively acute effect spanning days or weeks (generally reversible), the other occurring over a much longer time span (decades in humans) and that is self-sustaining and irreversible. The implications of these findings, as these authors emphasize in their concluding remarks, are that the various components of salt sensitivity (acute versus slow and reversible versus irreversible) should be considered in any comprehensive explanation of the effects of salt on blood pressure and in considering how best to reduce the impact of salt on health. This impact goes beyond the 'direct' effect of salt on blood pressure because, as mentioned above, high salt intake also drives obesity and cardiovascular risks by stimulating fluid intake that is often in the form of sugar-sweetened beverages or alcohol-containing (beer-like) beverages.

Furthermore, despite the general ban on the sales of alcoholic beverages to youth, teenagers are nonetheless increasingly exposed to the 'legal' consumption of alcohol contained in 'alcopops' - a term often used to describe flavoured alcoholic beverages to which various fruit juices or other flavourings have been added. They are considered as an initiation drink by many young people, and have been described by industry and concerned groups alike as 'bridging' or 'gateway' beverages for less experienced drinkers, who are not yet used to the taste of alcohol. As we are reminded by Suter and Schutz, alcohol has not only a higher energy value than sugars ( 7 versus $4 \mathrm{kcalg}^{-1}$ ), but its preferential utilization as a fuel substrate results in the suppression of fat oxidation, thereby conferring increased 
risk for weight gain, abdominal obesity and hypertriglyceridaemia. The focus of their review on the interactions between alcohol and exercise on postprandial lipidaemia and exercise performance is timely, as alcoholic beverages are often consumed before, during or after exercise sessions for a variety of reasons that include ergogenic, thirst quenching, rehydration or for social reasons.

\section{In search for solutions}

To wrap up this 4th Fribourg Obesity Research Conference meeting on the theme of dysfunctional foods with particular relevance to childhood and adolescence obesity and cardiovascular risks, Durrer-Schutz and Schutz present some encouraging (although preliminary) data suggesting that the implementation of holistic programmes in secondary schools - that include nutritional education combined with promotion of physical activity and behaviour modification - could constitute key factors in the prevention of youth obesity. They point out that each 'building block', as limited as it may be, can be useful to contribute to prevent the sharp rise in obesity in children and adolescents in Switzerland and elsewhere. Certainly, the European Union proposal to offer fruits and vegetables free to school children is another building block worth evaluating in the search for solutions to treat their obesigenic 'dysfunctional food' environment.

\section{Conflict of interest}

The authors have declared no financial interests.

AG Dulloo, Z Yang and JP Montani Department of Medicine, Division of Physiology, University of Fribourg, Fribourg, Switzerland E-mail: abdul.dulloo@unifr.ch 